\title{
Bayesian estimation of parametric uncertainties, quantification and reduction using optimal design of experiments for $\mathrm{CO}_{2}$ adsorption on amine sorbents
}

\author{
Jayashree Kalyanaraman, Yanfang Fan, Ying Labreche, Ryan P. Lively, \\ Yoshiaki Kawajiri, Matthew J. Realff ${ }^{1, *}$ \\ School of Chemical \& Biomolecular Engineering, Georgia Institute of Technology, 311 \\ Ferst Drive, Atlanta, GA 30332, United States
}

\begin{abstract}
Uncertainty quantification plays a significant role in establishing reliability of the mathematical models, while applying to process optimization or technology feasibility studies. Uncertainties, in general, could occur either in the mathematical model or in the model parameters. In this work, process of $\mathrm{CO}_{2}$ adsorption on amine sorbents loaded in hollow fibers is studied to quantify the impact of uncertainties in the adsorption isotherm parameters on the model prediction. The process design variable that is most closely related to the process economics is the $\mathrm{CO}_{2}$ sorption capacity, whose uncertainty is investigated. We apply Bayesian analysis and determine a utility function surface corresponding to the value of information gained by the respective experimental design point. It is demonstrated that performing an experiment at a condition with a higher utility has a higher reduction of design variable prediction uncertainty compared to choosing a design point at a lower utility.
\end{abstract}

\footnotetext{
${ }^{*}$ Corresponding author

Email address: matthew.realff@chbe.gatech.edu (Matthew J. Realff)

${ }^{1}$ Phone: 404-862-0440
} 
Keywords: Bayesian inference, adaptive metropolis, parallel propagation, optimal experimental design, $\mathrm{CO}_{2}$ adsorption

\section{Introduction}

Models play a key role in the design of chemical processes. Mathematical models, although meant to represent and predict system behavior accurately, are compromised due to the uncertainties of our knowledge of the system. Uncertainty in the model, and hence its predictions, arises either due to uncertainties associated with the model representation itself, causing model uncertainty or due to errors in the parameter values used in the model, leading to parametric uncertainty. Model uncertainty occurs primarily because of inaccurate understanding of the underlying system, or due to the oversimplifying assumptions used in the model while representing the system behavior. Parametric uncertainty, on the other hand, occurs due to the errors associated with the measurement variables or because of the sparsity of the available measurements used to estimate the model parameters. While it is theoretically possible to reduce or even completely remove the uncertainties in the model prediction, it can often be very challenging due to the inherent physical limitations associated with the measurement devices or the sheer complexity of performing an experiment to collect data. Nevertheless, it is important to quantify the uncertainties and their impact on the model predictions, in order to use the model reliably in process design.

Uncertainty Quantification (UQ) has grown in importance across many fields including chemical engineering (Duran and White, 1995; Coleman and Block, 2006; Hermanto et al. 2008; Najm et al. 2009; Angelikopoulous et 
al. 2012). There have been a number of methods developed to characterize, quantify, or propagate uncertainties including probabilistic methods, such as classical or frequentist inference and Bayesian inference (Omlin and Reichert, 1999), sampling methods such as Monte Carlo (Basil and Jamieson, 1998) and bootstrapping (Efron, 1979), and response surface methods (intrusive methods) such as polynomial chaos expansions (Xiu and Karniadakis, 2003) and Kriging approach (Yuan et al. 2008). Among these different methods, Bayesian inference offers several advantages over other methods as it does not require modification to the model and provides a comprehensive treatment of parametric and model uncertainties without any simplifying assumptions about their distributions (Omlin and Reichert, 1999; Alfaro et al. 2003). However, applying Bayesian inference and propagating the uncertainties using Monte Carlo sampling of the posterior parametric distribution or using polynomial chaos expansions leads to prohibitively expensive computation when applied to large models, as is commonly observed with any physio chemical process, involving coupled partial differential equations. In effect, most of the studies on quantifying uncertainties in the field of chemical engineering have been restricted to characterizing uncertainties (parametric inference problems), most of which involves simple batch system models such as kinetic models (Najm et al. 2009; Hsu et al. 2009; Albrecht, 2013), adsorption isotherms (Anagu et al. 2012; Mebane et al. 2013), fed batch fermentation reactors (Coleman and Block, 2006) and microbiology models (Pouillot et al. 2003), and only few studies have been performed on larger models such as packed bed adsorbers (Duran and White, 1995; CSTRs (Chen et al. 2004) and crystallization processes (Hermanto et al. 2008). 
While it is desirable to reduce the uncertainties associated with the model and parameters, it may often be difficult and expensive to perform experiments and collect data that are required to reduce the uncertainties. Under such scenarios, it is advantageous to determine the conditions at which performing experiments would provide maximum information gain. Such a strategy is termed as optimal experimental design (OED). By optimally designing the experiments, one can gain maximum amount of information about the system using the least number of experiments.

Model-based design of experimental methods using the Fisher Information Matrix (FIM) (Atkinson et al. 2007) have been well studied for models with linear parametric dependence, employing the well known optimal design conditions termed as alphabetical optimality criteria, which are evaluated as functionals of FIM (Franceschini and Macchietto, 2008). In case of models with nonlinear parametric dependence, a number of simplifying assumptions are made to employ FIM based optimal experimental design, including linearization of the model response and Gaussian approximation of the parametric distributions (Chu and Hahn, 2008). Bayesian experimental design, on the other hand, does not require, in general, (Chaloner and Verdinelli, 2009) any simplifying assumptions on the parametric distribution or the model linearization. Measure of the information gain using Bayesian analysis, is represented by an objective function called the utility function, which includes an optimality criteria which is maximised to determine the optimal design condition (Lindley, 1972). A detailed review on the Bayesian experimental design methods and the variations of the utility functions can be found in Chaloner and Verdinelli (2009). 
Bayesian experimental design was first introduced by Lindley (1972) where Shannon information gain is used from posterior to prior distribution for the experimental design followed by several others using variations of optimality criteria (Brooks, 1977, Shewry and Wynn, 1987). The computational expense of evaluating the utility function has been a major challenge in deploying the Bayesian design to determine the optimal experiment as most of the real world models are complex and cannot be analytically evaluated (Ryan, 2003; Terejanu et al. 2012). In effect, most of the reported work on Bayesian experimental design have either been using linear models and in the few studies having non-linear models, approximations of the utility function or Gaussian approximations of the posterior distributions are used (Russi et al. 2008; Mosbach et al. 2012). In that context, Muller and Parmigiani (1995) suggested using a Monte Carlo estimator and simulation based optimal design by fitting the Monte Carlo samples of utility surface.. However, they concluded that the evaluation becomes computationally prohibitive for large dimensions of design parameters. There have been very limited studies on Bayesian experimental design in the chemical engineering literature. In recent work, Solonen et al.(2012) applied the simulation based optimal design for a CSTR model using variance of predictions as the utility function. They sidestepped the computational complexity of posterior distribution evaluation after every added experiment, by weighing the parameters with the corresponding likelihood of the new measurements. Even with such methodology, the likelihood evaluation could turn out be computationally expensive for complex models. More recently, Huan et al. (2012) applied Bayesian D-optimality for experimental design involving a combustion kinetic model. 
They reduced the computational expense of likelihood evaluation by using a polynomial chaos surrogate and deployed noisy algorithms such as Simultaneous Perturbation Stochastic Approximation (SPSA) and Nelder-Mead Nonlinear Simplex (NMNS) requiring fewer evaluations of utility functions to arrive at the optimal design.

The objective of this work is therefore to perform Bayesian analysis and experimental design for a complex physio chemical process such as adsorption of $\mathrm{CO}_{2}$ from flue gas using amine sorbents loaded hollow fibers. Adsorption is a dynamic process and is described by an unsteady state model involving a number of coupled partial differential and algebraic equations. We apply Bayesian principle to quantify the impact of uncertainties in adsorption isotherm parameters on the prediction of a design variable, adsorption breakthrough capacity. Most of the studies on optimal design has been mostly towards reducing the uncertainties in parameters and there has been very few studies focused on reduction of prediction uncertainty, especially with Bayesian design (Solonen et al., 2012). In this work, we demonstrate the use of Bayesian optimal experimental design to identify the experimental condition which can provide the maximum reduction in the prediction uncertainty of the design variable of interest, the adsorption breakthrough capacity. There has been no reported study on the Bayesian analysis of uncertainty and reduction with optimal design of experiments for such a complex chemical process model as adsorption in a hollow fiber membrane to the best of authors' knowledge.

The rest of the paper is organized as follows. The application of interest in the work, adsorption of $\mathrm{CO}_{2}$ in the hollow fiber based amine sorbents is 
introduced in the following section along with the model formulation. It is followed by a brief introduction to the Bayesian framework used for uncertainty characterization, quantification and propagation, and the design of experiments based on the utility function evaluation. The next section describes the numerical framework utilized for the model implementation and the Bayesian analysis. The subsequent section discusses the results of the Bayesian inference, quantification and uncertainty reduction and the paper concludes with the summary and future tasks.

\section{Application: $\mathrm{CO}_{2}$ sorption in hollow fiber based sorbent}

There is an increased interest in $\mathrm{CO}_{2}$ removal from flue gas of coal fired power plants which has led to research into several new technologies (Yang et al. 2008). $\mathrm{CO}_{2}$ removal by adsorption process is promising in terms of relatively high energy efficiency and is being investigated extensively in the community with several variations in the operating configurations such as vacuum, pressure or temperature swing, and also with varied sorbents (Choi et al. 2009; Chou and Chen, 2004; Ebner et al. 2011). One of the configurations is to perform rapid thermal swing (RTSA) (Lively et al. 2009) of $\mathrm{CO}_{2}$ using amine sorbents supported in a hollow fiber configuration, which has been studied both experimentally and through models (Fan et al. (2014), Rezeai et al. (2014), Kalyanaraman et al. (2014). More details on the hollow fiber synthesis, amine sorbents used and the operating configuration can be found in Rezeai et al. (2013); Labreche et al. (2013). In the adsorption step of the four step cycle involved in the RTSA, flue gas containing $\mathrm{CO}_{2}$ flows in the shell side of the hollow fiber while the cooling water flows in the bore 
side removing the heat of adsorption. During the adsorption time period, $\mathrm{CO}_{2}$ from flue gas permeates through the hollow fiber and is adsorbed in the amine sorbents impregnated within the hollow fiber, thereby reducing the exit flue gas $\mathrm{CO}_{2}$ concentration to very low levels. In this work, we consider the $\mathrm{CO}_{2}$ adsorption in a hollow fiber that is post-infused with class 2 amine sorbents (Rezeai et al. 2013), wherein the silica support is grafted with aminopropyltrimethoxysilane (APS). Mathematical modeling of the RTSA process with hollow fiber based amine sorbents has already been developed and experimentally validated under various operating and process configurations (Kalyanaraman et al. 2014).

Schematic of a single hollow fiber with various dimensions used in the deployed model is described in Fig.1. We use a mass and heat transfer model similar to that reported in Kalyanaraman et al. (2014) for the study and is based on the experimental configuration reported in fan et al. (2014), where there is no water flow through the fiber bores. The heat of adsorption is rapidly removed by dissipation to the module wall and is modeled using the various heat sink sources as described in Kalyanaraman et al. (2014). We consider only the adsorption step of the RTSA process in this work and the mathematical model that is used in this work is based on the following simplifying assumptions.

- Radial gradients for the bulk gas phase concentration, velocity and temperature are neglected.

- Radial gradient in the fiber temperature is neglected.

- Pressure drop is assumed to be negligible and gas velocity is therefore 


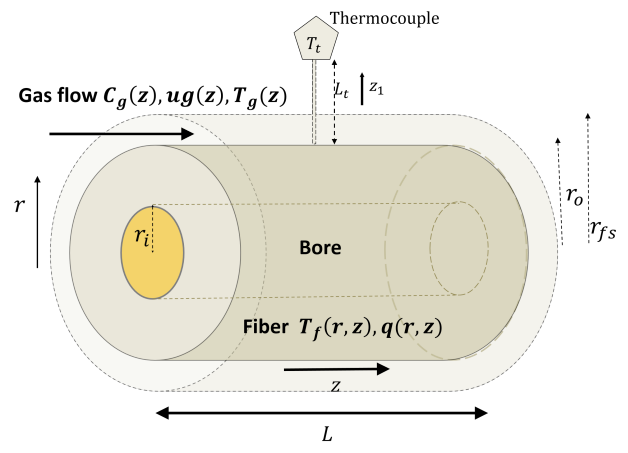

Figure 1: Schematic of a hollow fiber

assumed to be constant.

- Axial dispersion in the gas phase is neglected.

The component $\mathrm{CO}_{2}$ mass balance in the bulk gas phase is given by the following equation.

$$
\frac{\partial C_{g}}{\partial t}+u_{g} \frac{\partial C_{g}}{\partial z}+\frac{\partial q}{\partial t} \rho_{f}\left(1-\epsilon_{f}\right) \frac{r_{o}^{2}-r_{i}^{2}}{r_{f s}^{2}-r_{o}^{2}}=0
$$

where $C_{g}$ is the concentration of $\mathrm{CO}_{2}$ in the gas phase, $q$ is the $\mathrm{CO}_{2}$ concentration in the sorbent phase and $u_{g}$ is the gas velocity. Additionally, $r_{i}$ and $r_{o}$ are the fiber inner and outer radii and $r_{f s}$ is the free surface radius respectively. The boundary conditions of the mass balance equation at the fiber inlet $z=0$ and exit $z=L$ are given by $\left.C_{g}\right|_{z=0}=C_{i n}$ and $\left.\frac{\partial C_{g}}{\partial z}\right|_{z=L}=0$ respectively.

The sorbent phase $\mathrm{CO}_{2}$ component mass balance is based on the two site mass transfer model proposed and validated in Kalyanaraman et al. (2014) for the $\mathrm{CO}_{2}$ adsorption in amine sorbents and is described by the following 
equations.

$$
\begin{aligned}
\frac{\partial q_{\text {surface }}}{\partial t} & =K_{\text {ov,surface }}\left(q_{\text {eq }} \psi-q_{\text {surface }}\right) \\
\frac{\partial q_{\text {bulk }}}{\partial t} & =K_{\text {ov }, \text { bulk }}\left(q_{e q}(1-\psi)-q_{\text {bulk }}\right) \\
q(z, t) & =q_{\text {surface }}(z, t)+q_{b u l k}(z, t)
\end{aligned}
$$

where $q_{\text {surface }}$ and $q_{\text {bulk }}$ are the sorbent $\mathrm{CO}_{2}$ concentration in the surface and bulk amine sites respectively. The overall mass transfer coefficients are represented using $K_{o v, \text { surface }}$ and $K_{o v, b u l k}$ for the surface and bulk amine sites respectively. The fraction of the total amine available in the surface amine sites is given by the parameter $\psi$. Here, $q$ represents the total sorbent $\mathrm{CO}_{2}$ concentration and the average sorption capacity at any time $t$ in the hollow fiber is determined as follows.

$$
q_{q v g}(t)=\frac{1}{L} \int_{z=0}^{L} q(z, t) d z
$$

In Kalyanaraman et al. (2014), detailed model for the overall mass transfer coefficients $K_{o v, \text { surface }}$ and $K_{o v, b u l k}$ was developed. In this work, we use a simplified version of the model for the overall bulk mass transfer coefficients as follows.

$$
K_{o v, b u l k}=K_{b} e^{-\alpha q_{b u l k}}
$$

and $K_{\text {ov,surface }}$ is fixed at a constant value. In the above equation $K_{b}$ is the overall bulk mass transfer coefficient at the start of the adsorption process and the parameter $\alpha$ represents the decrease in the mass transfer coefficient with sorbent loading similar to the one used in Kalyanaraman et al. (2014). 
The above simplifications to the mass transfer model do not affect the study objective because the focus is on the impact of adsorption isotherm uncertainties on the breakthrough capacity.

The equilibrium $\mathrm{CO}_{2}$ capacity $q_{e q}$ at the given bulk gas concentration is defined using Toth adsorption isotherm as follows.

$$
q_{e q}=q_{m} \frac{b C_{g}}{\left(1+\left(b C_{g}\right)^{n}\right)^{1 / n}}
$$

where $q_{m}$ is the maximum adsorption capacity at a given temperature, $b$ is the adsorption affinity constant and $n$ is the heterogeneity parameter. The temperature dependency of these three parameters are defined as follows.

$$
\begin{gathered}
q_{m}=q_{m 0} e^{-\eta\left(1-\frac{T_{f}}{T_{0}}\right)} \\
\left.b=b_{0} e^{-\frac{\Delta H_{0}}{R_{g} T_{0}}\left(1-\frac{T_{0}}{T_{f}}\right.}\right) \\
n=A+B\left(1-\frac{T_{0}}{T_{f}}\right)
\end{gathered}
$$

where $q_{m 0}$ is the maximum sorption capacity at $T_{0}$ and $\eta$ is the parameter defining temperature dependency of $q_{m}$. Similarly $b_{0}$ is the affinity parameter at $T_{0}$ and $\Delta H_{0}$ is the isosteric heat of adsorption at zero loading. Finally, $A$ is the heterogeneity parameter at $T_{0}$ and $B$ defines the temperature dependency of $n$.

The performance of the adsorption process is typically quantified in terms of $\mathrm{CO}_{2}$ sorption capacity, i.e the amount of $\mathrm{CO}_{2}$ adsorbed in the sorbent from the gas phase untill a defined time known as the breakthrough time $t_{b r}$. Breakthrough time is defined as the time instant at which the $\mathrm{CO}_{2}$ concentration at the exit of the fiber module reaches a particular value of concentration, the breakthrough concentration $C_{b r}$. Adsorption breakthrough capacity $q_{b r}$ 
is therefore determined as follows:

$$
q_{b r}=\left.q_{a v g}(t)\right|_{t=t_{b r}}
$$

where $t_{b r}$ is determined by the following equation.

$$
t_{b r}=\left\{\max t \mid C_{g}(t, z=L) \leq C_{b r}\right\}
$$

The above equation determines the maximum time duration of the adsorption step such that the $\mathrm{CO}_{2}$ concentration at the fiber exit $\left(\mathrm{CO}_{2}\right.$ breakthrough concentration) does not exceed the maximum concentration $C_{b r}$.

Energy balance of the fiber phase is given in the following equation,

$$
\begin{array}{r}
\rho_{f} C_{p, f}\left(1-\epsilon_{f}\right) \frac{\partial T_{f}}{\partial t}-\lambda_{f} \frac{\partial^{2} T_{f}}{\partial z^{2}}-\frac{\partial q}{\partial t}\left(-\Delta H_{a d s}\right)\left(1-\epsilon_{f}\right) \rho_{f} \\
+h_{t}\left(\left.T_{f}\right|_{z=z_{t}}-\left.T_{t}\right|_{z_{1}=0}\right) \frac{A_{t}}{\left(r_{o}^{2}-r_{i}^{2}\right) L}+h_{g}\left(T_{f}(z)-T_{g}(z)\right) \frac{2 r_{o}}{\left(r_{o}^{2}-r_{i}^{2}\right)}=0
\end{array}
$$

where $\Delta H_{a d s}$ is the average isosteric heat of adsorption. The boundary conditions of $T_{f}$ are given by $\partial T_{f} /\left.\partial z\right|_{z=0}=\partial T_{f} /\left.\partial z\right|_{z=L}=0$. Here, $h_{t}$ and $h_{g}$ are the heat transfer coefficients of thermocouple and gas respectively. Additionally, the heat balance for the thermocouple is modeled using a one dimensional heat diffusion equation along the dimension $z^{\prime}$ and is given as follows.

$$
\rho_{t} C_{p, t} \frac{\partial T_{t}}{\partial t}-\lambda_{t} \frac{\partial^{2} T_{t}}{\partial z^{\prime 2}}=0
$$

The boundary conditions of the thermocouple temperature at $z^{\prime}=0$ and $z^{\prime}=L_{t}$ are given by $-\lambda_{t} \frac{\partial T_{t}}{\partial z^{\prime}}=h_{t}\left(\left.T_{f}\right|_{z=z_{t}}-\left.T_{t}\right|_{z^{\prime}=0}\right)$ and $\left.T_{t}\right|_{z^{\prime}=L_{t}}=T_{a m b}$ respectively. Finally, the energy balance of the gas phase is given as follows.

$$
\begin{aligned}
\rho_{g} C_{p, g}\left(\frac{\partial T_{g}}{\partial t}+u_{g} \frac{\partial T_{g}}{\partial z}\right) & +h_{g} \frac{2 r_{o}}{r_{f s}^{2}-r_{o}^{2}}\left(T_{g}(z)-T_{f}(z)\right) \\
& +U \frac{2 r_{f s}}{r_{f s}^{2}-r_{o D}^{2}}\left(T_{g}(z)-T_{a m b}\right)=0
\end{aligned}
$$


The boundary conditions for $T_{g}$ are $\left.T_{g}\right|_{z=0}=T_{g i n}$ and $\left.T_{g}\right|_{z=L}=T_{a m b}$.

\section{Bayesian framework}

In this work, Bayesian principle is applied for characterization, quantification and reduction of uncertainties using optimal design of experiments. Fig.2 compares the steps that are typically followed in a conventional approach of optimal design with the proposed approach. In the conventional approach (Fig.2a), the experimental design is focused towards reducing parametric uncertainty whereas in the proposed approach, the design criteria is the prediction variable uncertainty. The first step in the framework is to characterize the uncertainties, which is followed by quantification of their impact on the prediction variable. Optimal design is then determined as the experimental condition that has a maximum utility function, which is a measure of information gain of prediction variable. In reality, all the steps involved need to be repeated in a loop until the uncertainty of the prediction variable of interest is within the required threshold limits. In the results that follow, we demonstrate the framework by performing one iteration of the steps involved.

\subsection{Characterization of uncertainties}

Under the probabilistic framework, characterization of uncertainties, an inverse problem, is the first step towards uncertainty quantification. Uncertainties associated with adsorption isotherm parameters, denoted as $\theta=$ $\left(q_{m 0}, \eta, A, B, \ln b_{0}, \Delta H_{0}\right)$ are characterized using the measurements of equilibrium adsorption capacity $q_{e q}$. 


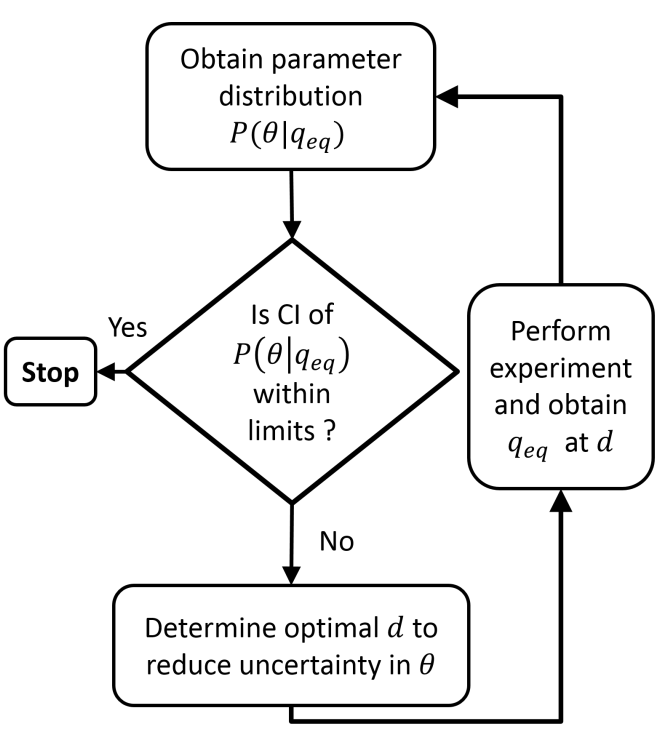

(a) Conventional approach

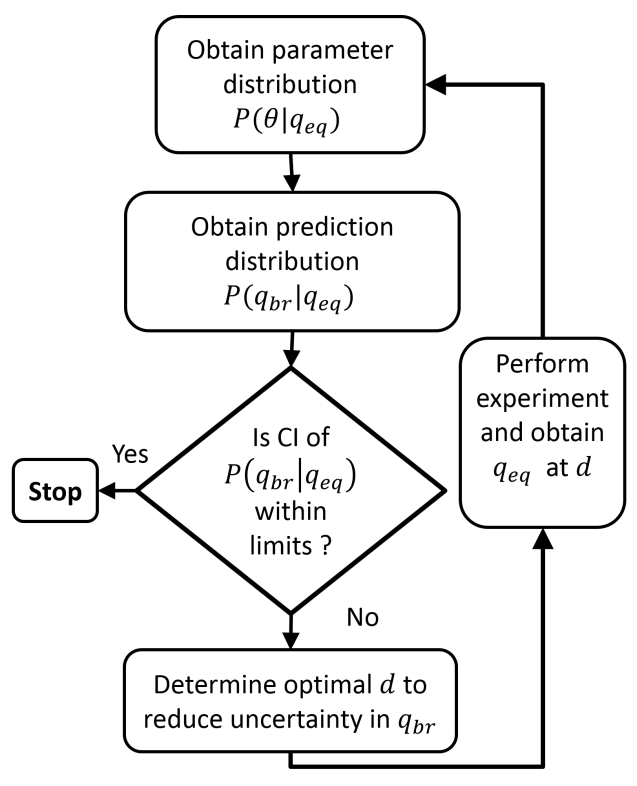

(b) Proposed approach

Figure 2: Steps involved in the parametric uncertainties 
Parametric uncertainties are characterized using the Bayesian principle, where the posterior distribution of parameters is related to the likelihood of observing a measurement variable, conditional on a given set of parameter values, as follows (Gelman et al. 2011).

$$
P\left(\Theta \mid q_{e q}\right)=\frac{\left.P\left(q_{e q} \mid \Theta\right) P(\Theta)\right)}{\int_{\Theta} \int_{q_{e q}} P\left(q_{e q} \mid \Theta\right) P(\Theta) d \Theta d q_{e q}}
$$

In the above equation, the adsorption isotherm parameters $\theta$ along with the hyperparameter $\sigma_{q_{e q}}$ are denoted as $\Theta=\left(\theta, \sigma_{q_{e q}}\right)$. Here, the hyperparameter $\sigma_{q_{e q}}$ is the the standard deviation of the measurement variable $q_{e q}$ which is characterized along with the main parameters using the hierarchical model approach. Hence, $P\left(\Theta \mid q_{e q}\right)$ is the posterior distribution of isotherm parameters and the hyperparameter and $P\left(q_{e q} \mid \Theta\right)$ is the likelihood of $q_{e q}$ given the isotherm parameters and hyperparameter, $\left(\theta, \sigma_{q_{e q}}\right)$ respectively. Accordingly, $P(\Theta)$ is the joint prior distribution of isotherm parameters and hyperparameter which is simply the product of the isotherm prior distributions and the hyperprior distribution which are independent of one another. The denominator in the above equation is the normalizing constant, called as the evidence parameter. Likelihood of observing $q_{e q}$ is assumed to follow a normal distribution with the mean, $q_{e q . m o d e l}$ and standard deviation, $\sigma_{q_{e} q}$ of measurement error, as follows.

$$
P\left(q_{e q} \mid \Theta\right)=\prod_{i}^{n} e^{\frac{\left(q_{e q, i}-q_{e q . m o d e l}(\theta), i\right.}{2 \sigma^{2}}}
$$

where $q_{\text {eq.model }(\theta), i}$ is determined from Eq.7 at a given set of adsorption isotherm parameters $\theta$. 


\subsection{Uncertainty Propagation}

The design variable, whose prediction uncertainty is of key interest, is the adsorption breakthrough capacity $q_{b r}$. The prediction distribution of $q_{b r}$ posterior to the measurement of $q_{e q}, P\left(q_{b r} \mid q_{e q}\right)$ is determined by marginalizing the conditional predictive distribution $P\left(q_{b r} \mid \Theta\right)$ over the parameters using the posterior parametric distribution $P\left(\Theta \mid q_{e q}\right)$ as follows.

$$
P\left(q_{b r} \mid q_{e q}\right)=\int_{\Theta} P\left(q_{b r} \mid \Theta\right) P\left(\Theta \mid q_{e q}\right) d \Theta
$$

It is to be noted that $q_{b r}$ and $q_{e q}$ are conditionally independent given $\Theta$, though $q_{b r}$ and $q_{e q}$ are actually dependent.

\subsection{Optimal experimental design}

The value of information gained by an experimental design point can be quantified using an objective function called the utility function, $U(d)$. As we are interested in reducing the uncertainty with respect to the prediction variable $q_{b r}$, the utility function is defined with respect to the information gain in the predictive distribution prior and posterior to performing an experiment at $d$. The measure of information gain is defined using the $\mathrm{K}-\mathrm{L}$ divergence as follows (Huan and Marzouk, 2012).

$$
U(d)=\int_{q_{e q}} \int_{q_{b r}} \log \frac{P\left(q_{b r} \mid q_{e q}, d\right)}{P\left(q_{b r}\right)} P\left(q_{b r} \mid q_{e q}, d\right) P\left(q_{e q} \mid d\right) d q_{b r} d q_{e q}
$$

where $d$ defines the experimental design condition, which in this case refers to the set of $[T, P]$ at which the adsorption equilibrium capacity $q_{e q}$ is measured. Additionally, $P\left(q_{b r} \mid q_{e q}, d\right)$ is the predictive distribution posterior to performing the experiment at $d$ and $P\left(q_{b r}\right)$ refers to the predictive distribution prior 
to performing the experiment. In the above formulation, the predictive distribution needs to be determined for several candidate design conditions, where each evaluation by itself involves determining the respective parametric distribution and running the model simulation defined in Eqns.(1)-(15) for every sample point in the respective parametric distribution. Such an evaluation is computationally very expensive.

The computational complexity involved can be significantly reduced by evaluating the utility function $U_{p}(d)$ as given below, where the Shannon information criteria utilizing the $\mathrm{K}-\mathrm{L}$ divergence is determined with respect to the parametric distribution rather than the predictive distribution as earlier (Eq.19), thereby eliminating the need to evaluate the predictive distribution.

$$
U_{p}(d)=\int_{q_{e q}} \int_{\Theta} \log \frac{P\left(\Theta \mid q_{e q}, d\right)}{P(\Theta)} \mid P\left(\Theta \mid q_{e q}, d\right) P\left(q_{e q} \mid d\right) d \Theta d q_{e q}
$$

However, our objective is to determine the experimental condition that maximizes the information gain with respect to the prediction variable rather than the parameters themselves. Therefore, we determine and isolate the parameters which have a higher sensitivity for the prediction variable and evaluate the information gain with respect to the sensitive parameters alone, while holding the other parameters at their nominal values as defined below.

$$
U^{\prime}(d)=\left.\int_{q_{e q}} \int_{\Theta} \log \frac{P\left(\Theta_{S} \mid q_{e q}, d\right)}{P\left(\Theta_{S}\right)}\right|_{\Theta_{N S}=\Theta_{\text {mean }}} P\left(\Theta \mid q_{e q}, d\right) P\left(q_{e q} \mid d\right) d \Theta d q_{e q}
$$

where $\Theta_{S}$ refers to the selective subset of parameters from $\Theta$ to which the design variable $q_{b r}$ is sensitive and $\Theta_{N S}$ refers to the remaining subset in $\Theta$.This modified utility function as defined in can effectively provide a measure of the information gain with respect to the prediction uncertainty. 


\subsection{Global Sensitivity Analysis}

Global Sensitivity Analysis (GSA) represents the sensitivity information with respect to the complete parametric space, in contrary to the local sensitivity analysis (LSA), which determine the sensitivity information at a local parametric value (Zhan et al. 2013).GSA is used especially when the parameters are correlated and have strong interactions with one another and/or vary over a large parameter space. We employ the Morris method, an one at a time (OAT) based screening method to determine the GSA of the adsorption isotherm parameters on the prediction variable (Campologno et al. 2007), (Iooss and Lemaitre, 2014).

In the OAT based Morris method, as the name suggests, each parameter is varied one at a time and the sensitivity of the respective parameter with respect to the output variable of interest is calculated. This local sensitivity is termed as an elementary effect (EE). Since the model output is a time dependent variable, a derived variable viz. the design variable $q_{b r}$ is used to determine the sensitivity values. In the notation used below, $\theta_{i, j}$ refers to $\mathrm{i}^{\text {th }}$ sample of $j^{\text {th }}$ parameter, in which the index $j$ refers to the members in set $\theta$ and $i$ refers to the row in the matrix domain $\Omega$, which is the complete set of parametric values. The size of the matrix domain $\Omega$ is of the size $($ row $\times$ column $)=(p \times n)$, which is representative of the joint parameter distribution. Here $p$ refers to the size of the set $\theta$ and $n$ refers to the number of samples which is representative of the joint parameter distribution. The elementary effect, $E E_{i, j}$ for the parameter $j$ with the $i^{\text {th }}$ set of values is determined as follows. 


$$
\begin{array}{r}
E E_{i, j}=\frac{q_{b r}\left(\theta_{i, 1}, . ., \theta_{i, j-1}, \theta_{\mathbf{i}+\mathbf{1}, \mathbf{j}}, \theta_{i, j+1}, . .\right)-q_{b r}\left(\theta_{i, 1}, . ., \theta_{i, j-1}, \theta_{\mathbf{i}, \mathbf{j}}, \theta_{i, j+1}, . .\right)}{\Delta \theta_{j}} \frac{\sigma_{\theta_{j}}}{\sigma_{q_{b r}}} \\
i=1 \ldots p ; j=1 \ldots n
\end{array}
$$

In the above equation $E E_{., j}$ is scaled using the standard deviation of $q_{b r}$ and $\theta_{j}$ for the numerator and denominator respectively (Sumner et al. 2012). To determine $E E_{i, j+1}$, all the $\theta_{j}$ are held at the previous values and only the $j+1^{\text {th }}$ parameter is incremented to $i+1^{\text {th }}$ value.

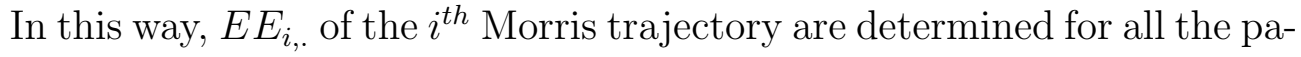
rameters $j=1 . . p$ and typically about $\mathrm{r}=20$ to 50 trajectories are evaluated, which are chosen randomly from the domain $\Omega$. The sensitivity information of the parameters is quantified using two measures, which are $\mu_{j}$ and $\sigma_{j}$ and are determined for every $j^{\text {th }}$ parameter. They are determined from the individual elementary effects as follows.

$$
\begin{array}{r}
\mu_{j}=\sum_{i=1}^{r} \frac{a b s\left(E E_{i, j}\right)}{r} \\
\sigma_{j}=\sqrt{\frac{\sum_{i=1}^{r}\left(E E_{i, j}-\mu_{j}\right)^{2}}{r-1}}
\end{array}
$$

\section{Experimental setup}

The adsorption equilibrium capacity $q_{e q}$ that is used to estimate the adsorption isotherm parametric uncertainty in this work were collected using thermo gravimetric analysis (TGA) experiments, which are performed at constant temperature $T$ and $\mathrm{CO}_{2}$ partial pressure $P$. Details on the TGA setup and the experimental conditions used can be found in Rezeai et al. (2013). 
The experimental data of $\mathrm{CO}_{2}$ breakthrough concentration profile and fiber temperature that are used for comparison with the prediction uncertainty were collected using the rapid thermal swing adsorption (RTSA) experimental setup. During the adsorption step of the experiment, a simulated flue gas of composition $14 \% \mathrm{CO}_{2}, 14 \% \mathrm{He}$ and the rest $\mathrm{N}_{2}$ was flown through a hollow fiber module and the composition of $\mathrm{CO}_{2}$ that exits the hollow fiber module, known as the breakthrough concentration profile was measured. Also, the fiber temperature at around the midpoint of the fiber module was measured to determine the rise in temperature due to heat of adsorption. Details of the RTSA setup and the experimental conditions can be found in Fan et al. (2014).

\section{Algorithm and Numerical framework}

This section describes the algorithm employed and the numerical implementation to solve the model and perform the uncertainty analysis. The complete framework which includes the numerical integration of model along with uncertainty analysis is implemented in Python and the computational expense is reduced by exploiting parallel computation wherever conditionally independent simulations can be performed.

\subsection{MCMC Simulation: Adaptive $M-H$ algorithm}

For complex models involving nonlinear likelihood distributions, solving Eq.16 to determine the posterior parametric distribution is not analytically tractable. In such cases, Markov Chain Monte Carlo(MCMC) simulation is the most commonly used efficient methodology to determine the posterior 
distribution of arbitrary complexity. Among the different algorithms to perform random walk MCMC simulation, Metropolis Hastings (M-H) algorithm is one of the most popular and simpler methods (Hastings, 1970). One of the primary performance measure that differentiates the effectiveness of the various MCMC algorithms is the speed at which the algorithm converges to the stationary posterior distribution, i.e equilibrium distribution which does not change any further with additional samples, especially when the number of parameters involved is large. As the conventional random walk $\mathrm{M}-\mathrm{H}$ algorithm has a slow rate of convergence primarily due to the difficulty involved in the choice of an appropriate proposal distribution covariance, adaptive Metroplis Hastings algorithm, which adaptively tunes the proposal covariance, is an effective method especially when dealing with a large number of parameters. Here, we use the adaptive Metropolis Hastings algorithm similar to the method of Atchade and Rosenthal(2005), where the proposal covariance is adaptively tuned depending on the continuous monitoring of the acceptance rate. Instead of tuning the covariance after every simulation, the adaptive tuning is performed at a frequency of $w$ simulations. A general description of the algorithm steps are briefly described as follows.

- Start the MCMC algorithm with an initial point $\theta_{0}$ and starting covariance of proposal distribution $\Sigma_{0}$.

- Choose a candidate point $\theta_{\text {cand }}$ from proposal distribution $q(\theta)=\mathcal{N}\left(\theta_{0}, \Sigma_{0}\right)$.

- Calculate the posterior $P\left(\theta_{\text {cand }} \mid q_{e q}\right)$ and $P\left(\theta_{0} \mid q_{e q}\right)$ using Eq.16 without evaluating the respective denominators and compute $\alpha=\min \left(\frac{P\left(\theta_{\text {cand }} \mid q_{e q}\right)}{P\left(\theta_{0} \mid q_{e q}\right)}, 1\right)$

- Generate $u \in \mathcal{U}(0,1)$ and accept $\theta_{\text {cand }}$ if $\alpha>u$, reject otherwise. 
- Compute acceptance rate, $r$ and repeat the following for every $w$ simulations and

if $r<0.23$ then $\Sigma_{\text {new }}=e_{d} \Sigma_{0}$

if $r>0.23$ then $\Sigma_{\text {new }}=s_{d} \Sigma_{0}$

Go back to step of choosing the candidate point with the modified proposal covariance $\Sigma_{\text {new }}$

In the above algorithm, notation $N$ refers to the multivariate Gaussian distribution, $\mathcal{U}$ refers to the uniform distribution and the reference value 0.23 (Atchade and Rosenthall, 2005) for the acceptance rate is the optimal acceptance rate required for a good mixed chain. In the actual implementation of the algorithm, additional tuning is performed depending upon the distance (number of simulations) between the two successive accepted samples. Moreover, as we have a hierarchical model with $\sigma_{q_{e q}}$ as a hyper parameter, it is also sampled during every simulation from a univariate Gaussian proposal distribution $\mathcal{N}(\mu, \sigma)$. Here $\mu$ and $\sigma$ are the mean and standard deviation of the hyperprior distribution, which is used as a proposal distribution for the hyperparameter. The multiplicative parameters $e_{d}$ and $s_{d}$ define the expanding and shrinking rate of the proposal covariance respectively. Depending on the current acceptance rate, the covariance is either shrunk or expanded by the respective multiplicative parameters. The values of the these two parameters along with $w$ are determined by tuning with trial and error runs until a faster convergence is obtained.

\subsection{Numerical integration of sorption model}

As the model involved is a system of coupled Partial Differential Algebraic Equations (PDAE), spatial discretisation is required in addition to time 
integration. As the gas phase equations are hyperbolic, a high resolution flux limiter based method is employed that helps to achieve high accuracy (less numerical diffusion) with a fewer number of finite elements. Monotonized Central-difference limiter (MC) (Leveque, 2004) proposed by van Leer is used for the convective fluxes involved in Eq.1-15 and Central Finite Difference (CFD) is used for the diffusive fluxes in Eq.13 and Eq.14. Time integration is performed using the inbuilt function scipy.integrate.odeint in Python documentation which switches between BDF and Adams depending upon the stiffness of the equation.

\subsection{Monte Carlo propagation in parallel}

As the Monte Carlo propagation of the posterior parametric distribution using Eq.18 involves independent simulations, parallelization using mpi is exploited to handle the otherwise prohibitively expensive computation. Therefore, the samples from the thinned posterior parametric distribution are propagated through the the model Eq.1-15 by running in parallel across multiple processors. The predictive distribution is thus determined using Monte Carlo integration as follows.

$$
\begin{aligned}
P\left(q_{b r} \mid q_{e q}\right)= & \int_{\Theta} P\left(q_{b r} \mid \Theta\right) P\left(\Theta \mid q_{e q}\right) d \Theta \\
& \approx \frac{1}{n_{m c m c}} \sum_{i=1}^{n_{m c m c}} P\left(q_{b r} \mid \Theta\right)
\end{aligned}
$$

where $\Theta=\left(\theta, \sigma_{q_{e q}}\right)$ are sampled from the posterior distribution $P\left(\Theta \mid q_{e q}\right)$ and $n_{m c m c}$ is the number of MCMC samples of the posterior distribution. 


\subsection{GSA evaluation}

Although GSA evaluation is less expensive compared to propagation, computing the sensitivity for $n=6$ parameters with $r=50$ trajectories involves at least 300 simulations of the model Eq.1-15 and is relatively expensive. Each trajectory is therefore run in parallel using mpi4py and the sensitivity information is collected from across processors to compute $\mu_{j}$ and $\sigma_{j}$

\subsection{Parallel computation of utility function}

The utility function in Eq.3.3 is evaluated using Monte Carlo estimation as follows.

$\left.U^{\prime}(d) \approx \frac{1}{n_{\text {obs }}} \frac{1}{n_{m c m c}} \sum_{i=1}^{n_{m c m c}} \sum_{j=1}^{n_{\text {obs }}}\left\{\log P\left(q_{e q, j} \mid \Theta_{S, i}, d\right)-\log P\left(q_{e q, j} \mid d\right)\right\}\right|_{\theta_{N S, i}=\theta_{\text {mean }}}$

In the above equation, the second term is evaluated as follows.

$$
\begin{aligned}
P\left(q_{e q, j} \mid d\right) & =\left.\int_{\Theta} P\left(q_{e q, j} \mid \Theta_{S}, d\right)\right|_{\Theta_{N S}=\Theta_{\text {mean }}} P\left(\Theta \mid q_{e q}\right) d \Theta \\
& \left.\approx \frac{1}{n_{m c m c}} \sum_{i=1}^{n_{m c m c}} P\left(q_{e q, j} \mid \Theta_{S, i}\right)\right|_{\Theta_{N S, i}=\Theta_{\text {mean }}}
\end{aligned}
$$

The optimal experimental design condition $d_{o p t}$ is determined by a complete enumeration of $U^{\prime}(d)$ over the search space of experimental conditions, which involve a two dimensional space of $(T, P)$. A $2 \mathrm{D}$ grid of $d \in(T, P)$ is designed with possible experimental conditions and $U^{\prime}(d)$ in Eq.3.3 is evaluated for each grid point. As evaluation of $U^{\prime}(d)$ at each grid point is independent of the other, each enumeration is performed in parallel across multiple processors. The design point with the highest value of $U^{\prime}(d)$ is the optimal condition $d_{\text {opt }}$ to perform the new experiment. 


\section{Results and Discussion}

This section describes the results for the uncertainty characterization, propagation and the optimal design of new experiment.

\subsection{Adsorption Isotherm Parametric Inference}

In this work, we study the uncertainties associated only with respect to the adsorption isotherm parameters $\theta$ due to the measurement uncertainties of the sorption equilibrium capacity $q_{e q}$. The rest of the model parameters viz. the mass transfer resistance and heat transfer parameters in the model are assumed to have deterministic values. The values of the assumed deterministic model parameters are determined by fitting the model prediction with the experimental observation of breakthrough concentration profile and fiber temperature, while holding the adsorption isotherm parameters at their nominal values. Thermo gravimetric (TGA) analysis experiments at various temperature and pressure $\left(\mathrm{CO}_{2}\right.$ concentration) were performed and the data were collected for the equilibrium sorption capacity $q_{e q}$ at the respective ex-

perimental conditions. Fig.3 shows the experimental data of $q_{e q}$ that is used to perform the Bayesian parametric inference.

As the adsorption equilibrium capacity $q_{b r}$ is conditionally independent of $q_{e q}$ given the isotherm parameters $\theta$, the model involving $q_{e q}$ and the isotherm parameters in Eq.7 alone can be used to characterize the isotherm parametric uncertainties. On the other hand, characterizing the mass transfer and heat transfer parameters would involve the complete PDAE model and performing the MCMC simulation, a serial computation for the complex PDAE system is prohibitive and other inference techniques such as using Polynomial Chaos 
expansion needs to be investigated for such problems (Marzouk et al., 2007). Since the main focus of the work is optimal design using the Bayesian principle to reduce the prediction uncertainty rather than a complete characterization of the parametric uncertainties in a complex physiochemical process as adsorption, we isolated a set of model parameters, the isotherm parameters for which the prediction variable $q_{b r}$ is highly sensitive and demonstrated the approach.

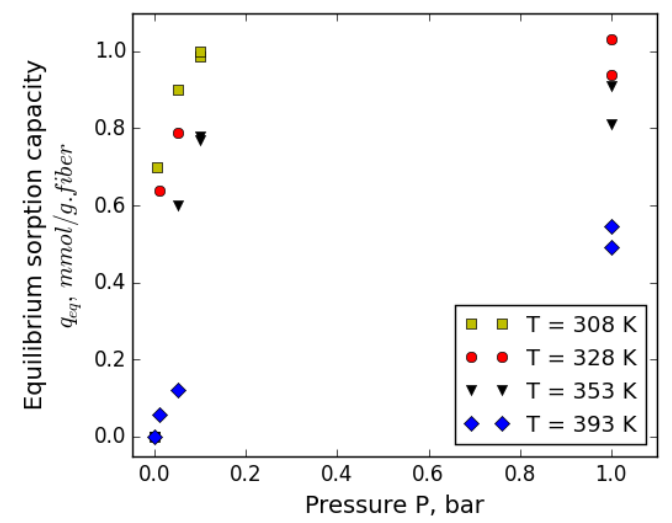

Figure 3: Experimental data of adsorption equilibrium capacity $q_{e q}$ at various temperature $T$ and $\mathrm{CO}_{2}$ pressure $P$

Specification of prior distribution is a key advantage of Bayesian reasoning as it allows the incorporatation of prior beliefs about the parameters in the estimation. However, an appropriate choice of the prior is required to avoid bias in the posterior distribution estimate, especially when there is insufficient data available. A good discussion on the role of priors and the different types of principle based priors can be found in Agostini (2003). With respect to the adsorption isotherm parameters, it is possible to obtain a good 
Table 1: Values of prior and hyperprior parameters

\begin{tabular}{lll}
\hline Parameters & Upper bound & Lower bound \\
\hline$q_{m 0}[\mathrm{mmol} / \mathrm{g}$ fiber $]$ & 0.1 & 10.0 \\
$\eta[-]$ & -5.0 & 5.0 \\
$A[-]$ & 0.0 & 2.0 \\
$B[-]$ & -6.0 & 10.0 \\
$\ln b_{0}\left[\mathrm{~Pa}^{-1}\right]$ & -9.0 & 9.0 \\
$-\Delta H_{0}\left[\times 10^{2} \mathrm{~kJ} / \mathrm{mol}\right]$ & 0.2 & 1.75 \\
\hline Hyperparameter & $\mu$ & $\sigma$ \\
$\sigma_{q_{e q}[-]}$ & 0.036 & 0.011 \\
\hline
\end{tabular}

estimate of their nominal values based on a nonlinear parameter estimation technique utilizing the available data. Based on the available measurement errors of $q_{e q}$ and their sensitivity with respect to adsorption parameters, we believe that the isotherm parameters can have a wide variation around the nominal values. Therefore, it is reasonable to assume either a Gaussian prior distribution with its mean at the obtained estimates and a large variance or an uniform distribution with a sufficiently larger bounds. In this work, we assumed the latter for an ease of implementation. For the measurement error hyperparameter $\sigma_{q_{e q}}$, we assume a Gaussian hyper prior with a constant mean as the availabe error measurements did not have any correlation with $q_{e q}$. The respective hyperparameters $\mu_{\sigma}, \sigma_{s q}$ are determined by performing an MCMC simulation using the few available repeat measurements (Fig.3). Table.1 shows the prior bounds of the isotherm parameters used and the parameters of the hyperprior distribution.

Two independent MCMC simulations starting from different initial points and employing adaptive $\mathrm{M}-\mathrm{H}$ algorithm are run in parallel for around 2.5 hours of CPU time while collecting about $4 \mathrm{M}$ samples. Fig.4 shows the 

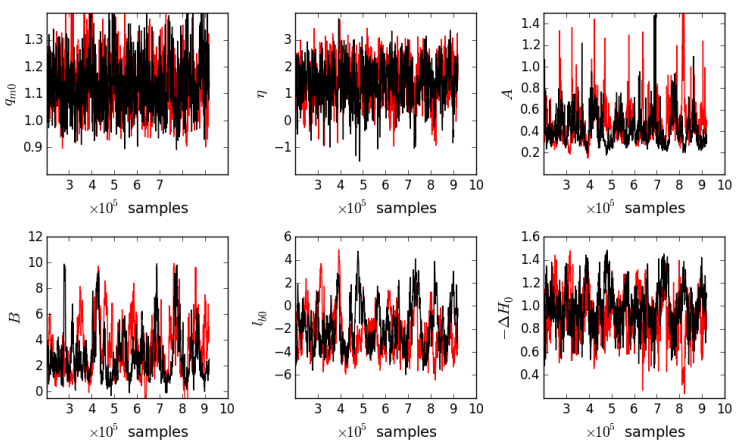

Figure 4: Trace of the posterior parametric distribution of the adsorption isotherm parameters

trace of two independent MCMC chains for all the adsorption isotherm parameters, wherein the orginal chain of length $4 \mathrm{M}$ samples is 'thinned' out every 650 samples after discarding nearly $0.2 \mathrm{M}$ samples for the "burnin" period. From the figure, it can also be observed that the two chains mix very well with respect to all the parameters and are converged to a constant mean along with a uniform spread around the mean. The autocorrelation of the resulting thinned distribution is also monitored for the sample independence and is shown in Appendix, which indicates that the samples are relatively uncorrelated.

Fig.5 shows the marginal posterior distribution of all the adsorption isotherm parameters and the hyperparameter. Marginal posterior distribution for a parameter $q_{m 0}, P\left(q_{m 0} \mid q_{e q}\right)$ for example, is determined from the joint posterior parametric distribution $P\left(\Theta \mid q_{e q}\right)$ by integrating out the other parameters from the joint distribution except $q_{m 0}$ as follows.

$$
P\left(q_{m 0} \mid q_{e q}\right)=\int_{\theta_{j}} P\left(\Theta \mid q_{e q}\right) d \theta_{j}, \theta_{j}=\left\{\eta, A, B, \ln b_{0}, \Delta H_{0}, \sigma_{q_{e q}}\right\}
$$



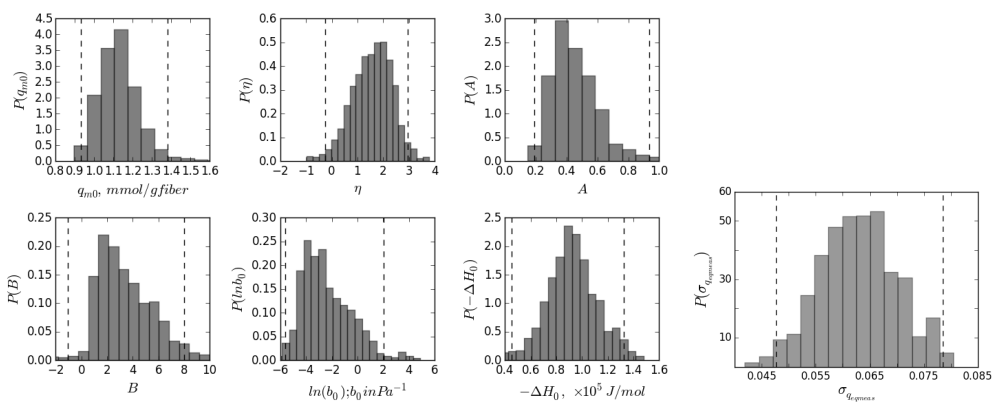

(a) Adsorption isotherm parameters

(b) Hyperparameter

Figure 5: Posterior parametric distribution of the adsorption isotherm parameters and hyperparameter $P\left(\Theta \mid q_{e q}\right) ; 95 \%$ credible interval shown by the dashed lines

\subsection{UQ propagation: posterior predictive distribution of the breakthrough capacity}

Once the adsorption isotherm parametric uncertainties are characterized, the impact of their uncertainties on the design variable of interest, the adsorption breakthrough capacity $q_{b r}$, is quantified by propagating the posterior parametric distribution $P\left(\Theta \mid q_{e q}\right)$ through the model defined by Eq.1-15 and evaluated using Eq.18. Table.2 lists the values that are used for all the properties and parameters in the model.

Figs.6(a),(b) and (c) show the distributions of model predictions, viz. $\mathrm{CO}_{2}$ breakthrough profile, fiber temperature measured at $z=3.5 \mathrm{in}$ and average sorption capacity $q_{a v g}$, resulting from the uncertainties associated with the adsorption isotherm parameters. The response distributions are plotted along with the experimental profiles of $\mathrm{CO}_{2}$ breakthrough and the fiber 
Table 2: Values of parameters and properties (deterministic parameters)

\begin{tabular}{|c|c|c|}
\hline Parameters & Values & Units \\
\hline$A_{t}$ & $6.40 \times 10^{-7}$ & $m^{2}$ \\
\hline$C_{p f}$ & 1200 & $J / k g K$ \\
\hline$C_{p g}$ & 1094 & $J / k g K$ \\
\hline$C_{p t}$ & 500 & $J / k g K$ \\
\hline$C_{g 0}$ & 0.00 & $\mathrm{~mol} / \mathrm{m}^{3}$ \\
\hline$C_{g, i n}$ & 5.40 & $\mathrm{~mol} / \mathrm{m}^{3}$ \\
\hline$h_{g}$ & 40.0 & $W / m^{2} K$ \\
\hline$h_{t}$ & 2500 & $W / m^{2} K$ \\
\hline$K_{o v, \text { surface }}$ & 1.00 & $s^{-1}$ \\
\hline$K_{o v, b}$ & 0.30 & $s^{-1}$ \\
\hline$L$ & 0.25 & $m$ \\
\hline$L_{t}$ & 0.05 & $m$ \\
\hline$r_{i}$ & 225 & $\mu m$ \\
\hline$r_{o}$ & 375 & $\mu m$ \\
\hline$r_{f s}$ & 809 & $\mu m$ \\
\hline$u_{g}$ & 0.07 & $\mathrm{~m} / \mathrm{s}$ \\
\hline$U$ & 60.0 & $W / m^{2} K$ \\
\hline$\alpha$ & 5.1 & g. fiber $/ \mathrm{mmol}$ \\
\hline$\epsilon_{f}$ & 0.48 & \\
\hline$\Delta H_{a d s}$ & -65.0 & $k J / m o l$ \\
\hline$\psi$ & 0.11 & \\
\hline$\rho_{f}$ & 960 & $\mathrm{~kg} / \mathrm{m}^{3}$ \\
\hline$\rho_{g}$ & 1.20 & $\mathrm{~kg} / \mathrm{m}^{3}$ \\
\hline
\end{tabular}



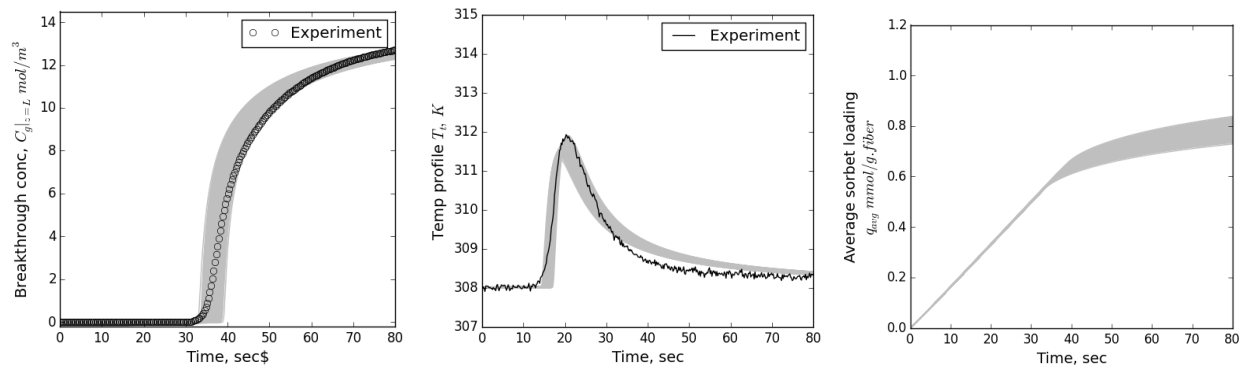

(a) Breakthrough curve

(b) Fiber temperature $T_{f}$

(c) Average sorption ca$C_{g} \mid z=L$ prediction prediction pacity $q_{\text {avg }}$ prediction

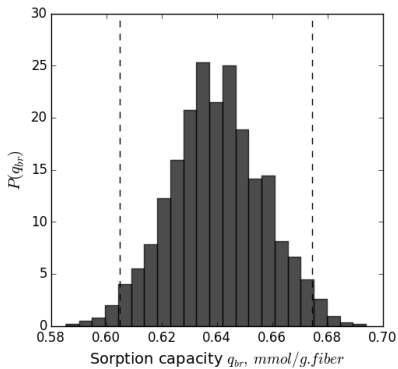

(d) Predictive distribution

of breakthrough capacity

$q_{b r}$

Figure 6: Distributions of model prediction variables due to adsorption isotherm parametric uncertainties 
temperature for comparison. The slight mismatch in the shape of the experimental $\mathrm{CO}_{2}$ breakthrough profile with the general trend of the model profile is due to the uncertainties that are associated with the mass transfer resistance parameters, which are not considered in this study. The uncertainty bandwidth of the breakthrough profile is expected to be much wider and with varying slopes in the presence of uncertainties with respect to the mass transfer parameters. Similarly, in the presence of uncertainties in heat transfer parameters, the thermal peak shape and height is also expected to have uncertainty in addition to what is observed now due to just isotherm parametric uncertainties.

The design variable $q_{b r}$ is calculated using Eq.11 where the adsorption capacity $q_{a v g}$ is considered till the time instant $t_{b r}$, as defined by Eq.12. For this study, we considered the breakthrough time $t_{b r}$ as the instant at which the concentration of $\mathrm{CO}_{2}$ at the exit reaches $50 \%$ of the feed concentration $C_{b r}=0.5 C_{i n}$. Shown in Fig.6(d) is the distribution of $q_{b r}$ along with the $95 \%$ credible interval(CI), which shows that the maximum achievable adsorption capacity without exceeding the breakthrough concentration $C_{b r}$ on the $\mathrm{CO}_{2}$ composition at the exit with $95 \%$ confidence is $0.605 \mathrm{mmol} / \mathrm{g}$. fiber. $95 \%$ credible interval (CI) $\left[q_{b r l}, q_{b r u}\right]$ for the prediction variable $q_{b r}$ can expressed mathematically as follows:

$$
P\left(q_{b r l}<q_{b r}<q_{b r u}\right)=0.95
$$

Parametric uncertainty propagation to obtain the predictive distribution was performed in parallel using 70 processors over a duration of 14 hours. 
Table 3: Global sensitivity values of adsorption isotherm parameters

\begin{tabular}{lll}
\hline Parameters & GSA mean $\mu_{j}$ & GSA $\sigma_{j}$ \\
$q_{m 0}$ & 2.07 & 0.33 \\
$\eta$ & 0.09 & 0.03 \\
$A$ & 6.04 & 4.17 \\
$B$ & 0.42 & 0.37 \\
$\ln b_{0}$ & 2.03 & 0.90 \\
$-\Delta H_{0}$ & 0.05 & 0.04 \\
\hline
\end{tabular}

\subsection{Optimal experimental design to reduce uncertainties}

As the first step towards evaluating the utility function defined in Eq.26, the GSA values for the adsorption isotherm parameters are determined by employing around $r=50$ trajectories and the resulting parameters $\mu_{j}$ and $\sigma_{j}$ are listed in Table.3. While a high value of $\mu_{j}$ indicates a high sensitivity of $q_{b r}$ with respect to that parameter $j$, a higher value of $\sigma_{j}$ is indicative of the nonlinear impact or a strong interaction of that parameter with other parameters. Based on the values of $\mu_{j}$ listed in Table. 3 from the GSA analysis, it can be found that $q_{b r}$ is highly sensitive with respect to the parameters $q_{m 0}, A$ and $\ln b_{0}$ having a high $\mu_{j}$ value and are denoted as $\theta_{S}$, while $q_{b r}$ is relatively insensitive with respect to the other three parameters and are denoted as $\theta_{N S}$ in Eq.26. Also, based on $\sigma_{j}$ values, $A$ is found to have a strong nonlinear impact or a strong interaction with other parameters. Running the 50 trajectories in parallel across 50 processors took nearly 9 hours of CPU time, as some of the combinations of isotherm parameters make the time integration stiff and time consuming.

Having identified $\Theta_{S}$, the utility function $U^{\prime}(d)$ is then evaluated using Eq.26 for a sequence of design conditions $d$ defined using the grid surface of 
dimensions $T \times P=12 \times 12$. Computation of $U^{\prime}(d)$ is also performed in parallel across 72 processors over a CPU time of 40 mins.

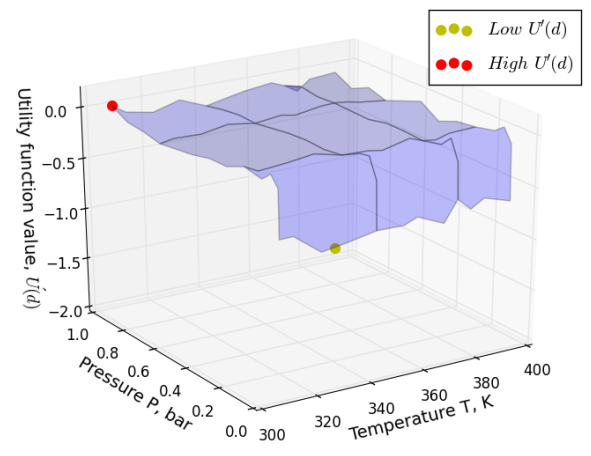

Figure 7: Utility function surface $U^{\prime}(d)$ over the design space of $[T, P]$

From the utility function surface $U(d)$ plotted in Fig.7, it can be seen that except the design condition at very low pressures around 0.005 bar, all other pressure and temperature values seem to have nearly same amount of potential information gain. This can be explained by the assumption that the measurement error quantified by $\sigma_{q_{e q}}$ is constant, i.e independent of the magnitude of $q_{e q}$. Consequently, an experimental data at low pressures of 0.005 bar can have a very minimal information gain due to low value of $q_{e q}$ at low pressures (Fig.3 and Eq.7) with a relatively larger error. On the other hand, at higher pressures, $q_{e q}$ has a relatively larger value and can have a potential information gain because of the smaller measurement error.

To verify and validate that the relative information gain at different design points as indicated by the utility function surface, two cases are studied each of which adds experimental data $q_{e q}$ at low utility and high utility values $U^{\prime}(d)$ respectively. A design point with a high utility function value is chosen at $\left(35^{\circ}\right.$ 
Table 4: Comparison of credible intervals between the two cases of $U(d)$ for all the parameters

\begin{tabular}{lll}
\hline Parameters & 95\% CI for high $U^{\prime}(d)$ & $\mathbf{9 5 \%}$ CI for low $U^{\prime}(d)$ \\
\hline$q_{m 0}$ & {$[1.03,1.36]$} & {$[0.93,1.38]$} \\
$\eta$ & {$[0.75,3.09]$} & {$[-0.25,2.95]$} \\
$A$ & {$[0.3,0.73]$} & {$[0.19,0.93]$} \\
$B$ & {$[0.75,8.5]$} & {$[-1.04,7.98]$} \\
$\ln b_{0}$ & {$[-5.30,0.24]$} & {$[-5.59,2.05]$} \\
$-\Delta H_{0}$ & {$[0.55,1.5]$} & {$[0.45,1.32]$} \\
$\sigma_{q_{e q}}$ & {$[0.05,0.08]$} & {$[0.05,0.08]$} \\
\hline
\end{tabular}

C, 1 bar) and an other case with a low utility function value is chosen at $\left(55^{\circ}\right.$ C, 0.005 bar). TGA experiments are peformed at both the design conditions $\left(55^{\circ} \mathrm{C}, 0.005 \mathrm{bar}\right)$ and $\left(35^{\circ} \mathrm{C}, 1 \mathrm{bar}\right)$ and the $q_{e q}$ values are found as 0.56 $\mathrm{mmol} / \mathrm{g}$. fiber and $0.91 \mathrm{mmol} / \mathrm{g}$. fiber respectively. MCMC simulations are performed for both the cases and the posterior distribution of the isotherm parameters are obtained and are compared against one another in Fig.8.
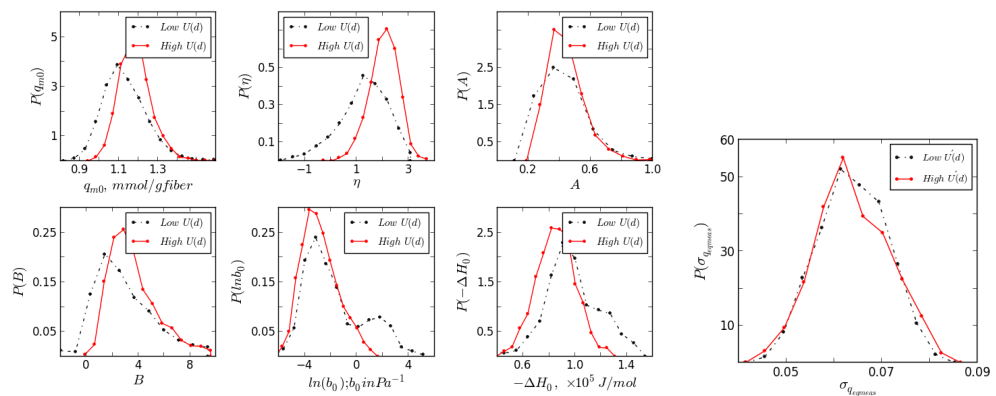
(a) Adsorption isotherm parameters
(b) Hyperparameter

Figure 8: Comparison of the posterior distribution of parameters $P\left(\Theta \mid q_{e q}, d\right)$ between the two cases of adding a data corresponding to high and low $U(d)$ respectively

The 95\% credible intervals (CI) are compared for the two cases for all the 
parameters in Table.4. As seen from the table, the case where a data point is added at higher $U^{\prime}(d)$ has a smaller uncertainty or narrower CI for almost all the adsorption isotherm parameters compared to the case where a data point is added at a lower utility value. The hyperparameter $\sigma_{q_{e q}}$, however, shows no significant difference in the uncertainty range, which indicates that the measurement error by itself has not been reduced, partly due to the fact that we assume a constant standard deviation of measurement error.

With the posterior parametric distribution for the two cases, the respective predictive distributions for the breakthrough profiles and $q_{b r}$, which are of key interest, are determined and compared in Figs.9 and Fig.10 respectively. From the figures, it can be clearly seen that the addition of an experiment at the high utility function reduces the uncertainty in breakthrough profile distribution and hence in $q_{b r}$ more than the case with the experimental data added corresponding to the lower utility function value. Based on the $95 \%$ credible intervals shown of $P\left(q_{b r}\right)$, the maximum sorption capacity that can be obtained without violating the breakthrough concentration constraint of $\mathrm{CO}_{2}$ at the exit is increased from the value of $0.605 \mathrm{mmol} / \mathrm{g}$. fiber in the base case (Fig.6(d)) to $0.63 \mathrm{mmol} / \mathrm{g}$.fiber (Fig.9), around 5\% increase with an addition of a single experimental point at a higher utility value. On the other hand, the addition of an experimental point at a lower utility value did not make any significant difference in the uncertainty compared to the base case. To give an economic perspective of the gain due to uncertainty reduction, Kulkarni and Sholl showed that an increase in the adsorption breakthrough capacity by $25 \%$ can result in the reduction of the net cost of $\mathrm{CO}_{2}$ capture by $23 \%$ (reduction from approximately $110 \$ / \mathrm{tCO}_{2}$ to $90 \$ / \mathrm{tCO}_{2}$ ). 

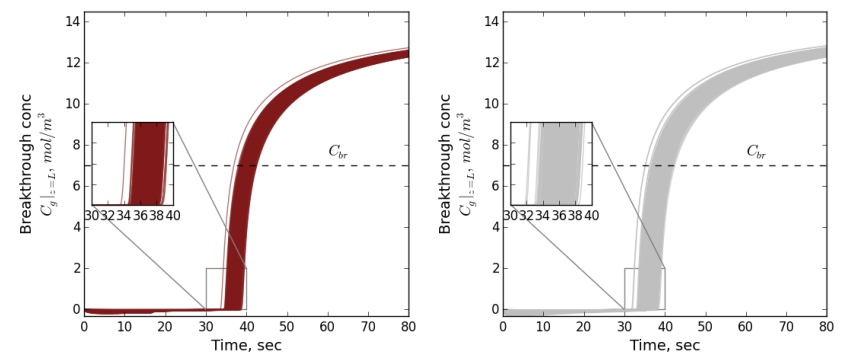

(a) An experiment at high (b) An experiment at low $U^{\prime}(d)$ added $\quad U^{\prime}(d)$ added

Figure 9: Comparison of the breakthrough profile distribution between the two cases of added experimental data at different utility $U^{\prime}(d)$ values

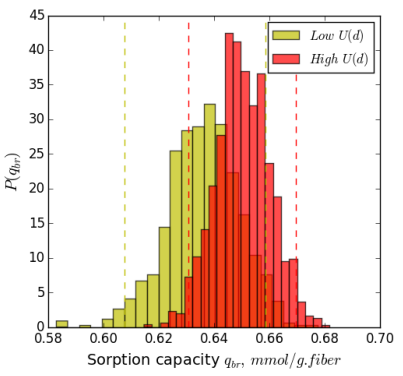

Figure 10: Comparison of the predictive distribution between the two cases with the corresponding dashed lines bounding 95\% CI 


\section{Conclusion and Future work}

A comprehensive Bayesian treatment of parametric uncertainties, including the characterization, propagation and reduction using optimal experimental design is applied for a complex physio chemical process of $\mathrm{CO}_{2}$ adsorption on amine sorbent loaded hollow fibers. The uncertainties associated with the adsorption isotherm parameters that occur due to the measurement errors of sorption equilibrium capacity $q_{e q}$ are investigated to assess their impact on the prediction design variable, the average $\mathrm{CO}_{2}$ sorption capacity at the breakthrough time. Adaptive Metropolis Hastings algorithm is employed to perform MCMC simulation of six adsorption isotherm parameters $\theta$ in addition to the hierarchical update of a hyperparameter, the measurement standard deviation $\sigma_{q_{e q}}$. The posterior parametric distribution is then propagated through the complex hollow fiber sorption model to determine the distribution in the prediction of adsorption capacity. The computational complexity in propagating the posterior parametric distribution using MCMC sampling of the posterior distribution is handled by parallelizing the propagation across multiple processors, in addition to using a high resolution MC method that effectively reduces the number of finite elements required in PDE discretization of mass and energy balance.

The final aspect of uncertainty quantification, which is to optimally design the experiments to reduce the uncertainty, was also addressed. To do so, we used a modified utility function that is defined using the joint likelihood distribution with respect to the selected parameters for which the prediction design variable $q_{b r}$ is highly sensitive. The defined utility function, a measure of information gain with respect to uncertainty reduction of prediction design 
variable, was evaluated using a simulation based design approach to determine the optimal experimental condition. Again, a complete parallelization was exploited to minimize computation time, which was already reduced to some extent by modifying the utility function for the design variable prediction and expressing it in terms of the distributions over parameters. The validity of the approach was demonstrated by adding an experimental data at the two extreme cases of utility function. It was found that the addition of an experimental data at the higher utility had a significantly higher reduction of uncertainty in the prediction of sorption capacity $q_{b r}$ than adding data at a lower utility. The future efforts may include the extension of the total Bayesian framework to include the uncertainties involved in the other important parameters of the sorption process, namely the mass transfer resistance parameters.

\section{Acknowledgement}

The authors would like to thank the U.S Department of Energy for supporting this work (Grant number DE-FE007804). However, any opinions,

findings or recommendations expressed herein are those of the author(s) and do not neccessarily reflect the views of the DOE. 


\title{
9. Nomenclature
}

\author{
$A, B \quad$ constants defining the temperature dependency of $n$ \\ $b \quad$ Toth isotherm affinity constant $\left[\mathrm{m}^{3} / \mathrm{mol}\right]$ \\ $b_{0} \quad$ parameter defining the temperature dependency of $b\left[\mathrm{~m}^{3} / \mathrm{mol}\right]$ \\ $C_{b r} \quad$ Limiting $\mathrm{CO}_{2}$ gas phase concentration at breakthrough $\left[\mathrm{mol} / \mathrm{m}^{3}\right]$ \\ $C_{g} \quad \mathrm{CO}_{2}$ gas phase concentration $\left[\mathrm{mol} / \mathrm{m}^{3}\right]$ \\ $C_{p} \quad$ Specific heat capacity of fiber $[\mathrm{J} / \mathrm{kgK}]$ \\ $e_{d} \quad$ Multiplicative factor defining the expansion rate of proposal covariance matrix \\ $h_{g} \quad$ Flue gas convective heat transfer coefficient $\left[\mathrm{W} / \mathrm{m}^{2} \mathrm{~K}\right]$ \\ $h_{t} \quad$ Convective heat transfer coefficient between thermocouple and module $\left[\mathrm{W} / \mathrm{m}^{2} \mathrm{~K}\right]$ \\ L Length $[\mathrm{m}]$ \\ n Toth isotherm heterogeneity parameter \\ $\mathcal{N} \quad$ Normal distribution \\ $P \quad$ Pressure $[\mathrm{Pa}]$ \\ $q_{\text {avg }} \quad$ Average $\mathrm{CO}_{2}$ loading in sorbent [mmol/gfiber] \\ $q_{\text {bulk }} \quad \mathrm{CO}_{2}$ loading in sorbent within the bulk PEI sites [mmol/gfiber] \\ $q_{b r} \quad \mathrm{CO}_{2}$ loading in sorbent at breakthrough time $t_{b r}[\mathrm{mmol} / \mathrm{gfiber}]$ \\ $q_{\text {eq }} \quad \mathrm{CO}_{2}$ loading at equilibrium $[\mathrm{mmol} / \mathrm{gfiber}]$ \\ $q_{m} \quad$ Maximum possible $\mathrm{CO}_{2}$ loading at a given temperature [mmol/gfiber] \\ $q_{m 0} \quad$ Maximum possible $\mathrm{CO}_{2}$ loading at the reference temperature $T_{0}$ [mmol/gfiber] \\ $q_{\text {surface }} \quad \mathrm{CO}_{2}$ loading in sorbent within the surface PEI sites[mmol/gfiber] \\ $r_{o} \quad$ outer fiber radius $[\mathrm{m}]$ \\ $r_{i} \quad$ inner fiber radius $[\mathrm{m}]$ \\ $r_{f s} \quad$ radius of free space surrounding fiber $[\mathrm{m}]$ \\ $s_{d} \quad$ Multiplicative factor defining the shrinking rate of proposal covariance matrix \\ $T \quad$ Temperature $[\mathrm{K}]$ \\ $u_{g} \quad$ Bulk gas velocity $[\mathrm{m} / \mathrm{s}]$ \\ $U(d), U^{\prime}(d)$ Utility function describing the information gain of experiment at $d$ \\ $U \quad$ Overall heat transfer coefficient of the module $\left[\mathrm{W} / \mathrm{m}^{2} \mathrm{~K}\right]$ \\ $\mathcal{U} \quad$ Uniform distribution \\ $1 / K_{o v, \text { surface }}$ Overall mass transfer resistance for $\mathrm{CO}_{2}$ adsorption on surface PEI sites [s] \\ $1 / K_{\text {ov bulk }}$ Overall mass transfer resistance for $\mathrm{CO}_{2}$ adsorption on bulk PEI sites [s]
}


Greek symbols

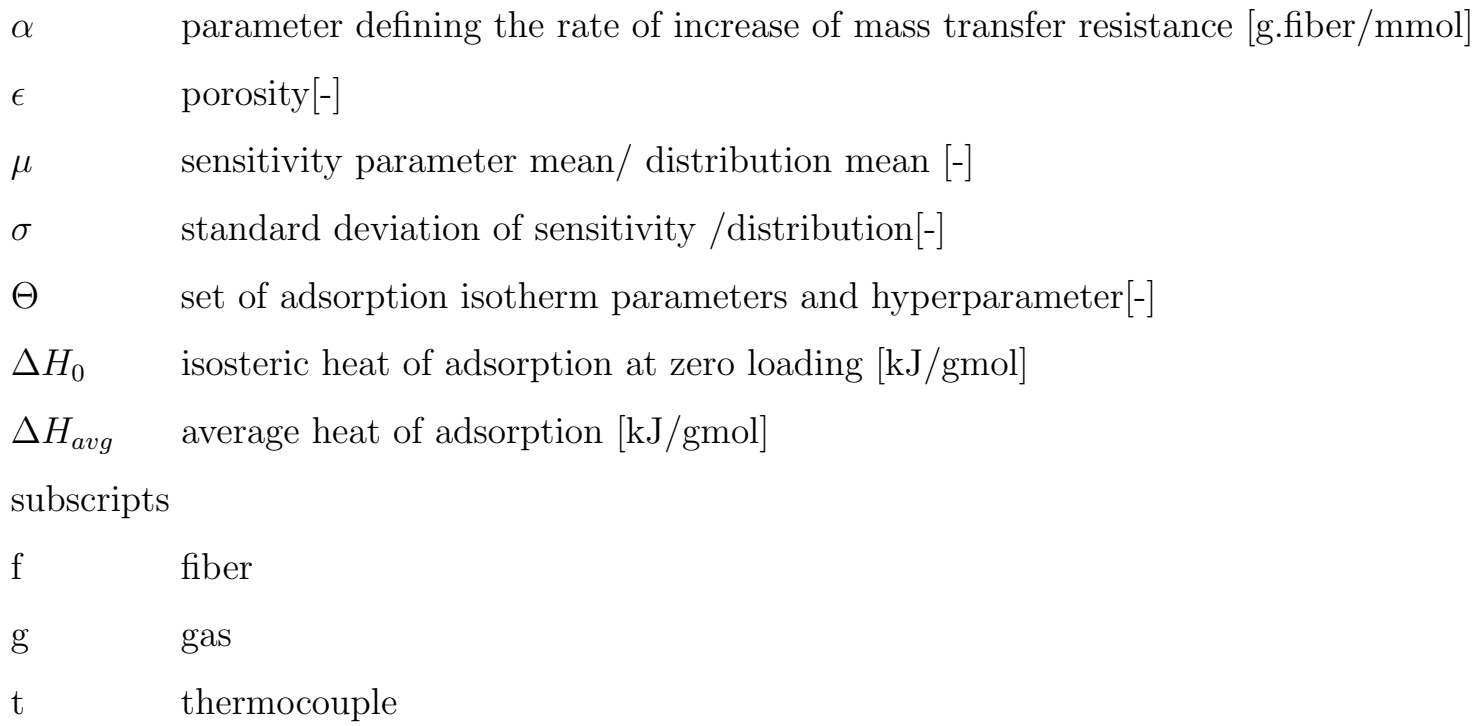

\section{Appendix A.}
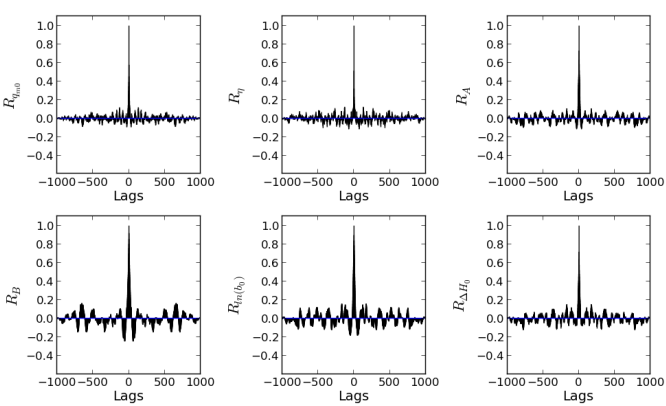

Figure A.1: Autocorrelation $R(\theta)$ (Gelman et al. 2011) of posterior parametric distribution samples 


\section{References}

[1] Duran MA, White BS. Bayesian estimation applied to effective heat transfer coefficients in a packed bed. Chemical Engg Science 1995;50:495510.

[2] Chen WS, Bakshi BR, Goel PK, Ungarala S. Bayesian rectification by sequential Monte Carlo sampling- Unconstrained nonlinear dynamic systems. Ind Eng Chem Res 2004;43(14):4012-4025.

[3] Coleman MC, Block DE. Bayesian parameter estimation with informative priors for nonlinear systems. AIChE J 2006;52:651-667.

[4] Hermanto MW, Kee NC, Tan RBH, Chen MS, Braatz RD. Robust Bayesian estimation of kinetics for the polymorphic transformation of L-glutamic acid crystals. AIChE J 2008;58:3248-3259.

[5] Najm HN, Debusschere BJ, Marzouk YM, Widdmer S, Le Maitre OP. Uncertainty quantification in chemical systems. International J. for Numerical Methods in Engg 2009;80:789-814.

[6] Hsu SH, Stamatis SD, Caruthers JM, Delgass WN, Venkatasubramanian $\mathrm{V}$ Bayesian framework for building kinetic models of catalytic systems. Ind Eng Chem Res 2009;48:4768-4790.

[7] Angelikopoulos P, Papadimitriou C, Koumoutsakos P. Bayesian uncertainty quantification and propagation in molecular dynamics simulations: A high performance computing framework. Journal of Chemical Physics 2012;137:144103 
[8] Omlin M, Reichert P. A comparison of techniques for the estimation of model prediction uncertainty. Ecological Modeling 1999;115:45-59.

[9] Basil M, Jamieson AW. Uncertainty of complex systems by Monte Carlo simulation. $16^{\text {th }}$ North Sea Flow Measurement Workshop, Gleneagles:1998

[10] Efron B. Bootstrap methods: another look at the jackknife. Annals of Statistics 1979;7:1-26.

[11] Xiu D, Karniadakis GE. Modeling uncertainty in flow simulations via generalized polynomial chaos. Journal of Computational Physics 2003;187:137-167.

[12] Yuan J, Wang K, Yu T, Fang M. Reliable multi-objective optimization of high speed WEDM process based on Gaussian process regression. Int J of Machine Tools and Manufacture 2008;48:47-60.

[13] Alfaro M, Zoller S, Lutzoni F. Bayes or bootstrap? A simulation study comparing the performance of Bayesian Markov chain Monte Carlo sampling and bootstrapping in assessing phylogenetic confidence. Molecular Biology and Evolution 2003;20(2):255-266.

[14] Albrecht J. Estimating reaction model parameter uncertainty with Markov chain Monte Carlo. Computers and Chemical Engg 2013;48:1428.

[15] Anagu I, Ingwesen J, Iden SC, Utermann J, Durner W, Streck T. Geoderma 2012;173-174:42-29. 
[16] Mebane DS, Bhat KS, Kress JD, Fauth DJ, Gray ML, Lee A, Miller DC. Bayesian calibration of thermodynamic models for the uptake of $\mathrm{CO}_{2}$ in supported amine sorbents using ab initio priors. Physical Chemistry Chemical Physics 2013;15(12):4355-4366.

[17] Pouillot R, Albert I, Cornu M, Denos JB. Estimation of uncertainty and variability in bacterial growth using Bayesian inference. Application to listeria monocytogenes. Int J Food Microbiology 2003;81:87-104

[18] Atkinson AC, Donev AN, Tobias RD. Optimum experimental designs. With SAS New York: Oxford University Press;2007

[19] Franceschini G, Macchietto S. Model based design of experiments for parameter precision:state of the art. Chemical Engg Sciences 2008;63:48464872 .

[20] Brooks S, Gelman A, Jones GL, Meng X. Handbook of Markov Chain Monte Carlo: Chapman and Hall;2011

[21] Chu R, Hahn J. Parameter set selection for estimation of nonlinear dynamic systems. AIChE J. 2007;53(11):2858-2870.

[22] Chaloner K, Verdinelli I. Bayesian experimental design: a review. Statistical Science 1995;10(3):273-304.

[23] Lindley DV. Bayesian statistics-a review: SIAM, Philadelphia;1972.

[24] Brooks RJ. Optimal regression design for control in linear regression. Biometrika 1977;64:319-325. 
[25] Shewry MC, Wynn HP. Maximum entropy sampling. J Appl Statistics 1987;14:165-170.

[26] Ryan KJ. Estimating expected information gains for experimental designs with application to random fatigue-limit model. Journal of Computatiomal and Graphical Statistics 2003;12(3):585-603.

[27] Terejanu G, Upadhyay RR, Miki K. Bayesian experimental design for the active nitridation of graphite by atomic nitrogen. Experimental Thermal and Fluid Science 2012;36:178-193.

[28] Russi T, Packard A, Feeley R, Frenkack M. Sensitivity analysis of uncertainty in model prediction. Journal of Physical Chemistry A. 2008;112(12):2579-2588.

[29] Mosbach S, Braumann A, Man PLW, Kastner CA, Brownbridge GPE, Kraft M. Iterative improvement of Bayesian parameter estimates for an engine model by means of experimental design. Combustion and Flame 2012;159:13303-1313.

[30] Muller P, Parmigiani G. Optimal design via curve fitting of Monte Carlo experiments. Journal of Americal Stat. Assoc. 1995;90:1322-1330.

[31] Solonen A, Haario H, Laine M. Simulation-based optimal design using a response variance criterion. Journal of Computational and Graphical Statistics 2012;21(1):234-252.

[32] Huan X, Marzouk YM. Simulation-based optimal Bayesian experimental design for nonlinear systems. Journal of Computational Physics $2013 ; 232(1): 288-317$. 
[33] Yang H, Xu Z, Fan M, Gupta R, Slimane RB, Bland AE, Wright I. Progress in carbon dioxide separation and capture: A review. Journal of Environmental Science2008;20:14-27.

[34] Choi S, Drese JH, Jones CW. Adsorbent materials for carbon dioxide capture from large anthropogenic point sources. Chem Sus Chem 2009;2:796-854.

[35] Chou CT, Chen CY. Carbon dioxide recovery by vacuum swing adsorption. Separation and Purification Technology 2004;39(1-2):51-65.

[36] Enber AD, Gray ML, Chisholm NG, Black QT, Mumford DD, Nicholson MM, Ritter JA. Suitability of a solid amine sorbent for $\mathrm{CO}_{2}$ capture by pressure swing adsorption. Ind. and Engg. Chemistry Research 2011;50:5634-5641.

[37] Lively RR, Chance RR, Kelley BT, Deckman HW, Drese JH, Jones CW, Koros WJ. Hollow fiber adsorbents for $\mathrm{CO}_{2}$ removal from flue gas. Ind and Engg Chemistry Research 2009;48(15):7314-7324.

[38] Rezeai F, Lively RP, Labreche Y, Chen G, Fan Y, Koros WJ, Jones CW. Aminosilane-grafted polymer/silica hollow fiber adsorbents for $\mathrm{CO}_{2}$ capture from flue gas. Applied Materials and Interfaces 2013;5:3921-3931.

[39] Labreche Y, Lively RP, Rezeai F, Chen G, Jones CW, Koros WJ. Post-spinning infusion of poly(ethylenimine) into polymer/silica hollow fiber sorbents for carbon dioxide capture. Chemical Engg Journal 2013;221:166-175. 
[40] Fan Y, Lively RP, Labreche Y, Rezeai F, Koros WJ, Jones CW. Evaluation of $\mathrm{CO}_{2}$ adsorption dynamics of polymer/silica supported poly(ethylenimine hollow fiber sorbents in rapid thermal swing adsorption. Int Journal of Greenhouse Gas Control 2014;21:61-71.

[41] Rezeai F, Swernath S, Kalyanaraman J, Lively RP, Kawajiri Y, Realff MJ. Modeling of rapid temperature swing adsorption using hollow fiber sorbents.Chemical Engg. Science 2014;113: 62-76.

[42] Kalyanaraman J, Fan Y, Lively RP, Koros WJ, Jones CW, Realff MJ, Kawajiri, Y. Modeling and experimental validation of carbon dioxide sorption on hollow fibers loaded with silica-supported poly(ethylenimine). Chemical Engg Journal 2014; 259:737-751.

[43] Zhan C, Song X, Xia J, Tong C. An efficient integrated approach for global sensitivity analysis of hydrological model parameters. Environmental Modeling \& Software 2013;41:39-52.

[44] Iooss B, Lemaitre P. A review on global sensitivity analysis methods:2014.

[45] Campolongo F, Cariboni J, Saltelli, A. An effective screening design for sensitivity analysis of large models. Environmental Modelling \& Software $2007 ; 22: 1509-1518$.

[46] Sumner T, Shepherd E, Bogle, IDL. A methodology for global-sensitivity analysis of time-dependent outputs in systems biology modelling. Journal of Royal Soc. Interface 2012; 9(74):2156-66. 
[47] Python documentation. https://docs.python.org/2/

[48] Hastings, W. Monte Carlo sampling methods using Markov chains and their applications. Biometrika 1970; 57: 97-109.

[49] Atchade YF, Rosenthal JS.. On adaptive Markov chain Monte Carlo algorithms. Bernoulli 2005; 11: 815-828.

[50] Leveque, R. Finite-volume methods for hyperbolic problems: Cambridge University Press, 2004.

[51] Cowles MK, Carlin, BP. Markov Chain Monte Carlo Covergence diagnostics: A comparative review. Journal of the American Stat Association 1996; 91(434): 883-904.

[52] Agostini GD, Bayesian inference in processing experimental data principles and applications, Reports on Progress in Physics 2003; 66:13831420.

[53] Marzouk YM, Najm, HN, Rahn, LA. Stochastic spectral methods for efficient Bayesian solution of inverse problems Journal of Computational Physics 2007; 224: 560-586.

[54] Kulkarni AR, Sholl, DS. Analysis of equilibrium-based TSA processes for direct capture of $\mathrm{CO}_{2}$ from air Ind.Engg.Chem.Res, 2012;51(25):86318645. 